\title{
Magnetorheography; Calculation of Blood Flow from Surface Induced Potentials
}

\author{
Osamu OkaI, M.D.* and Masamitsu Oshrma, M.D.**
}

\section{SUMmary}

This research is an attempt to measure blood flow without doing any hurt to the body. When the body is placed in a high magnetic field the blood-flow-induced electromotive force (EMF) can be detected with the surface electrodes using the same principle as an electromagnetic flowmeter. This blood flow measurement is named magnetorheography (its recording: $M R G)$.

A theoretical analysis of the potential in the tissue shows that blood flow can be calculated from the surface-induced EMF (MRG) without measuring the radius and the depth of the vessel from the skin surface, when the surface is fairly flat in the vicinity of the vessel. A model experiment verified the theory.

In order to apply the theory to the measurement in an in situ situation, a structural coefficient was introduced which is related to the external shape, internal tissue compositions and their impedance of the body. Using such a coefficient the flow calculated from MRG, by an equation including the coefficient, agreed with the flow actually recorded.

In experiments on the thighs of 8 dogs weighing 7 to $17 \mathrm{Kg}, \mathrm{MRG}$ proved to be proportional to arterial flow and the coefficients were approximately constant. These results indicate that blood flow may possibly be estimated by the calculation from MRG.

\section{Additional Indexing Words :}

Arterial flow Venous flow Electromagnetic flowmeter High magnetic field Surface electrode Calculation of flow

$A$ vention. When a body was placed in a high magnetic field, the bloodflow-induced electromotive force was detected with a surface electrode by the same principle as for an electromagnetic flowmeter. ${ }^{2)-4), 6), 7)}$

We have attemped to calculate arterial flow from the surface-induced

* Department of Surgical Science, the Heart Institute of Japan, Tokyo Women's Medical College, 10 Kawada-cho, Shinjuku, Tokyo.

** Institute of Medical Electronics, Faculty of Medicine, University of Tokyo, Hongo, Bunkyo, Tokyo.

Present Address: Department of Physiology, Kyorin University School of Medicine, 6-20-2 Shinkawa, Mitaka, Tokyo 181, Japan.

Received for publication January 14, 1975. 
potentials detected with surface electrode in this paper.

This nonbleeding measurement of blood flow, using a high magnetic field, is named magnetorheography; the appratus, magnetorheograph; the record, magnetorheogram (MRG).

\section{Theoretical Consideration}

\section{Mathematical Description}

Faraday's law of electromagnetic induction states that when blood moves through a magnetic field, an electromotive force $(\mathrm{EMF})$ is generated in a direction mutually perpendicular to both the magnetic field and the direction of blood flow. In this study the surface induced EMF (MRG) was detected with the surface electrodes. Then MRG is treated for the mathematical description in appendix.

As shown in Fig. 1, MRG on the skin surface is given by the equation:

$$
\mathrm{E}=\frac{20}{\pi} \frac{\sigma_{1}}{\sigma_{1}+\sigma_{2}} \mathrm{QH} \frac{\mathrm{y}_{1}}{\mathrm{x}^{2}+\mathrm{y}_{1}^{2}}
$$

where $E$ is electrical potential of $\mathrm{MRG} ; \sigma_{1}$ and $\sigma_{2}$ electrical conductivities of blood and tissue, respectively; $x$, electrode distance (distance of a locating point of an electrode from a point $P_{0}$ on the skin surface just above the vessel, is named a vessel surface point); $y_{1}$, vessel depth (distance of a vessel $P_{\checkmark}$ from $P_{0}$ ); $Q$, flow; $\mathrm{H}$, magnetic field intensity.

Selective Measurement of Arterial Flow

When the signal $E$ is fed to an A-C amplifier with a time constant of $\mathrm{T}$ sec, its frequency transfer function $G(i \omega)$ is

$$
G(j \omega)=\frac{1}{1+\sqrt{\frac{1}{\omega^{2} T^{2}}}}
$$

where $\omega$ is an angular velocity. If the signal consists of very low frequency components and $\mathrm{D}-\mathrm{C}$ (venous flow), the function indicates

$$
G(j \omega) \ll 1
$$

If the signal consists of high frequency components (arterial flow), the function represents

$$
G(j \omega) \simeq 1
$$

From eqs. (2-1) and (2-2) it is suggested that only arterial flow signals (peakto-peak flow signals) can be picked up out of these 2 arterial and venous flow signals using an $\mathrm{A}-\mathrm{C}$ amplifier.

Calculation of Flow from $M R G$

On any part of the body surface which is fairly flat in the vicinity of the blood vessel, MRG may be mainly due to the dipole induced by blood flow and its image.

For calculation of a flow the next 3 methods were established by modifying 


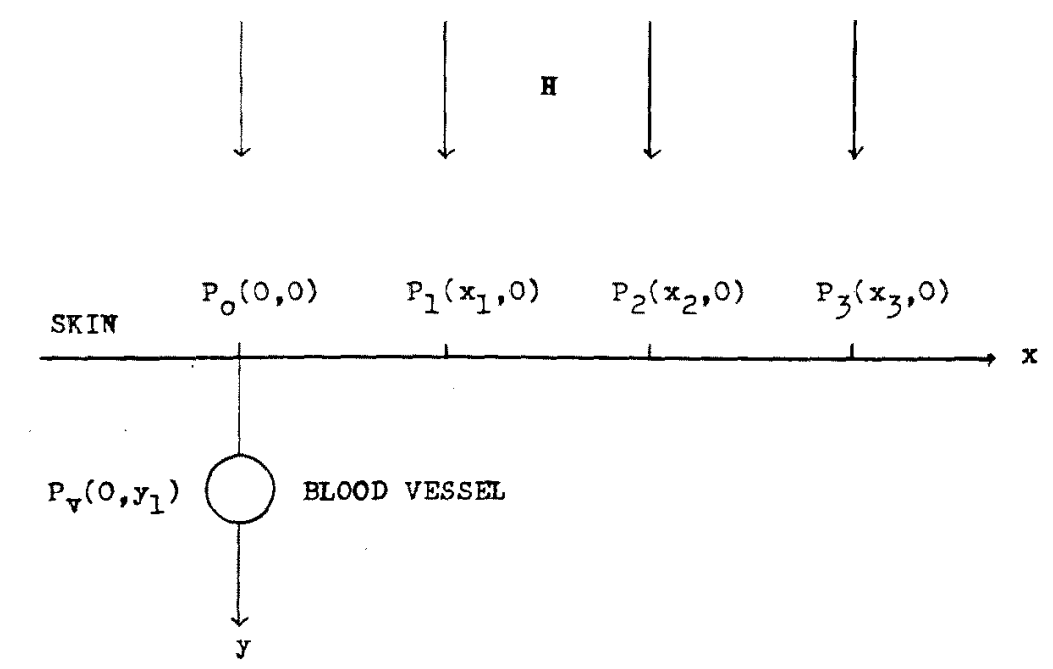

Fig. 1. Relationship among the skin surface, the vessel, and 3 measuring points $P_{1}, P_{2}$, and $P_{3}$ on a cross section, which are expressed in an orthogonal co-ordinate system, $\mathrm{O}-\mathrm{xy}$, with the vessel surface point as the origin $\mathrm{P}_{0}$, the skin surface as the $X$ co-ordinate, and the direction of the vessel depth as the $y$ co-ordinate; the $\mathrm{x}$ co-ordinate is perpendicular to both the axis of the vessel and the magnetic field $(\mathrm{H}) ; \mathrm{P}_{\mathrm{V}}$ is a center of the vessel on a cross section.

eq. (1). In this case the relationship among the skin surface, the vessel, and 3 measuring points $P_{1}, P_{2}$, and $P_{3}$ for an orthogonal co-ordinate system is shown in Fig. 1. Potentials $E_{1}, E_{2}$ and $E_{3}$ can be detected with these 3 surface electrodes which are placed at points $P_{1}, P_{2}$ and $P_{3}$, namely at electrode distances of $x_{1}, x_{2}$ and $x_{3}$ from the vessel surface point, respectively.

1) One electrode method

Measuring the vessel depth $y$ and the electrode distance $x$ by means of $x$ ray, ultrasonic facilities or others, we get a flow $Q$, by a rearrangement of eq. (1) and an introduction of flow coefficient $k^{\prime}$

$$
\mathrm{Q}=\frac{\mathrm{k}^{\prime}}{\mathrm{H}}\left(\mathrm{x}+\frac{\mathrm{y}^{2}}{\mathrm{x}}\right) \mathrm{E} \quad\left(\mathrm{k}^{\prime}=\frac{\pi}{20}-\frac{\sigma_{1}+\sigma_{2}}{\sigma_{1}}\right)
$$

where $E$ shows a potential $(\mathrm{mV}) ; H$, magnetic field intensity (K gauss); $Q$, flow $(\mathrm{ml} / \mathrm{sec}) ; x$, electrode distance $(\mathrm{cm}) ; y$, vessel depth $(\mathrm{cm})$.

2) Two electrode method

When it is difficult to measure the vessel depth, the use of two electrode method is applicable to the flow measurement. Two electrodes are placed at points $P_{1}$ and $P_{2}$, namely at electrode distances of $x_{1}$ and $x_{2}$, respectively. Eliminating $y$ from eq. (1) by substituting the potentials $E_{1}$ and $E_{\mathrm{g}}$ into eq. (1), we get

$$
\mathrm{Q}=\frac{\mathrm{k}^{\prime}}{\mathrm{H}} \frac{\mathrm{E}_{1} \mathrm{E}_{2}\left(\mathrm{x}_{2}^{2}-\mathrm{x}_{1}^{2}\right)}{\mathrm{E}_{1} \mathrm{E}_{2}-\mathrm{E}_{2} \mathrm{x}_{1}}
$$

3) Three electrode method

When it is difficult to measure the vessel depth and the vessel surface point, 
the use of three electrode method is applicable. Placing 3 electrodes at points of $P_{1}, P_{2}$ and $P_{3}$, namely at electrode distances of $x_{1}, x_{2}$ and $x_{3}$, respectively, the detected potentials $E_{1}, E_{2}$ and $E_{3}$ were substituted into eq. (1). In this case, let $x_{2}=x_{1}+a$, $x_{3}=x_{1}+a+b$, the second order equation is obtainable by eliminating $x_{1}$ and $y_{1}$ as follows:

$$
\begin{aligned}
M^{2} b E_{1}-(a+b) E_{2}+a E_{3}-M\left(a^{2}\right. & +2 a b) E_{1} E_{2}-(2 a b+b) E_{2} E_{3} \\
& +\left(b^{2}-a^{2}\right) E_{1} E_{3}+2 a b(a+b) E_{1} E_{2} E_{3}=0
\end{aligned}
$$

where $M=H Q \mid k^{\prime} ; a, b$, constants.

In practice, let $a, b=1$ for simplicity, we express eq. (3-3) by rearranging it as follows:

$$
M^{2}\left(E_{1}-2 E_{2}+E_{3}\right)-3 M\left(E_{1}-E_{3}\right) E_{2}+4 E_{1} E_{2} E_{3}=0
$$

Of the 2 roots, the one is selected which gives the best approximation to the flow calculated by the two electrode method. Then we get

$$
Q=\frac{k^{\prime}}{H} M
$$

\section{Model Experiment}

\section{Relationship between MRG and the Radius of the Vessel}

As shown in Fig. 2, a central coaxial tube with 2 radii of 5 (A) and $2.5 \mathrm{~mm}(\mathrm{~B})$ was bored in a box-shaped model of starch gel, which was placed in a high magnetic field $(H)$ of $1 \mathrm{~K}$ gauss. Two pairs of electrodes $\left(\mathrm{S}_{1}\right.$ and $\mathrm{S}_{2}$ ) were placed on both sides of the model.

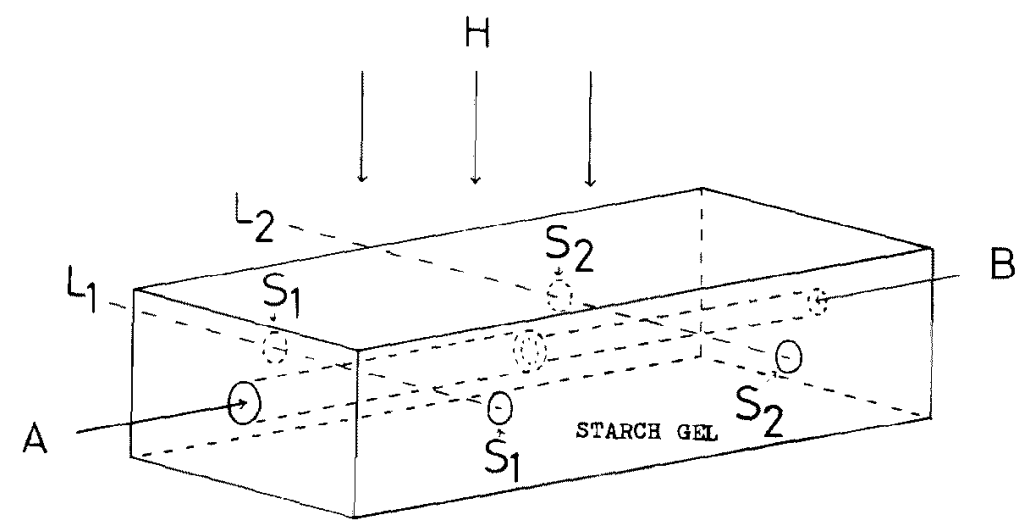

Fig. 2. Schematic presentation of model of starch gel for examining effect of the radius of a vessel on flow-induced potentials; 2 pairs of electrodes $\left(S_{1}\right.$ and $\left.S_{2}\right)$ are placed on both sides of the model; broken lines connecting 2 electrodes $\left(L_{1}\right.$ and $L_{2}$ ) cross the axis of the coaxial tube (A and $B$ ) with different radii of 5 and $2.5 \mathrm{~mm}$ respectively; the 2 lines are also perpendicular to both axis of the tube and the magnetic field $(\mathrm{H})$. 
MRG
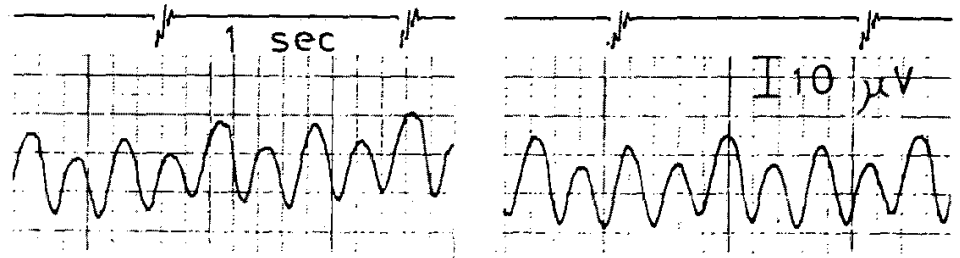

F L

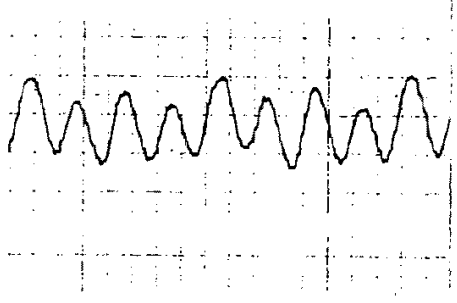

A

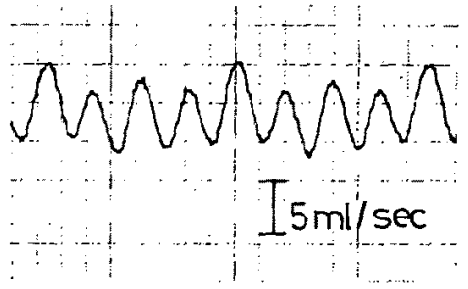

B

Fig. 3. Potentials (MRG) induced by the flows through the coaxial tube with 2 radii of 5 (A) and $2.5 \mathrm{~mm}$ (B); FL are flows obtained by an electromagnetic flowmeter.

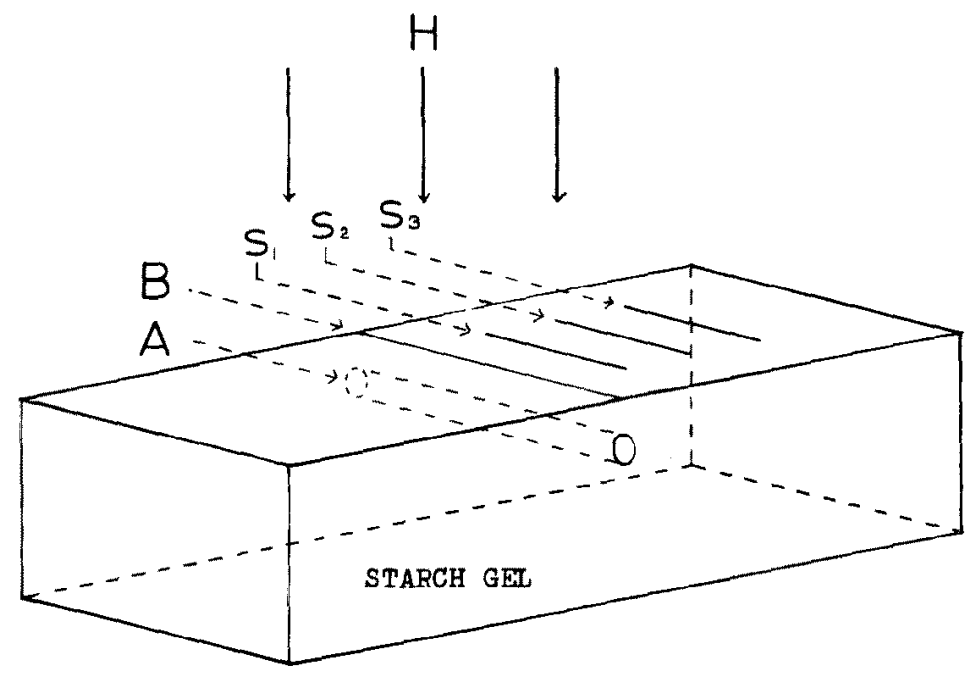

Fig. 4. Schematic presentation of a model of starch gel for calculating flows from flow-induced potentials; 3 needle-shape surface electrodes $\left\langle S_{1}, S_{2}\right.$, and $\left.\mathrm{S}_{3}\right)$ are placed at electrode distances of $1\left(\mathrm{~B}-\mathrm{S}_{1}\right), 2\left(\mathrm{~B}-\mathrm{S}_{2}\right)$, and $3 \mathrm{~cm}$ $\left(B-S_{3}\right)$, to make the axis of the electrodes paralleled to the axis of the tube; a reference electrodes is placed on the line (B); a tube (A) is bored at a distance of $1 \mathrm{~cm}$ from the surface, to make the axis of the tube paralleled to the line (B). 
Pulsatile flow using saline was given to the tube by a roller pump, and the flow-induced potentials (MRG) were fed to an A-C amplifier with a time constant of $1.5 \mathrm{sec}$.

MRG (A and B) induced by flows through the coaxial tube with radii of 5 and $2.5 \mathrm{~mm}$ respectively, are shown in Fig. 3. In spite of the difference in radii of the tube, no significant difference between $M R G$ for the 2 different radii was noted, suggesting that the $M R G$ were related only to a flow, and not influenced by the radius of the tube. This result verified eq. (1).

\section{Validation of Flow Calculation}

These 3 methods were applied to the model experiment using a boxshaped model of starch gel. As shown in Fig. 4, a tube (A) with a radius of $2 \mathrm{~mm}$ was bored in the model at a distance of $1 \mathrm{~cm}$ from the surface, to make the axis of the tube paralleled to the line $(B)$ on the surface. Three surface electrodes $\left(\mathrm{S}_{1}, \mathrm{~S}_{2}\right.$, and $\left.\mathrm{S}_{3}\right)$ were placed on the surface at electrode distances of 1,2 , and $3 \mathrm{~cm}$, respectively.

Pulsatile flows using saline were given to the tube by a roller pump, and the flow-induced signals (MRG) were fed to an A-C amplifier with a time constant of $1.5 \mathrm{sec}$. Flow patterns are shown in Fig. 5.

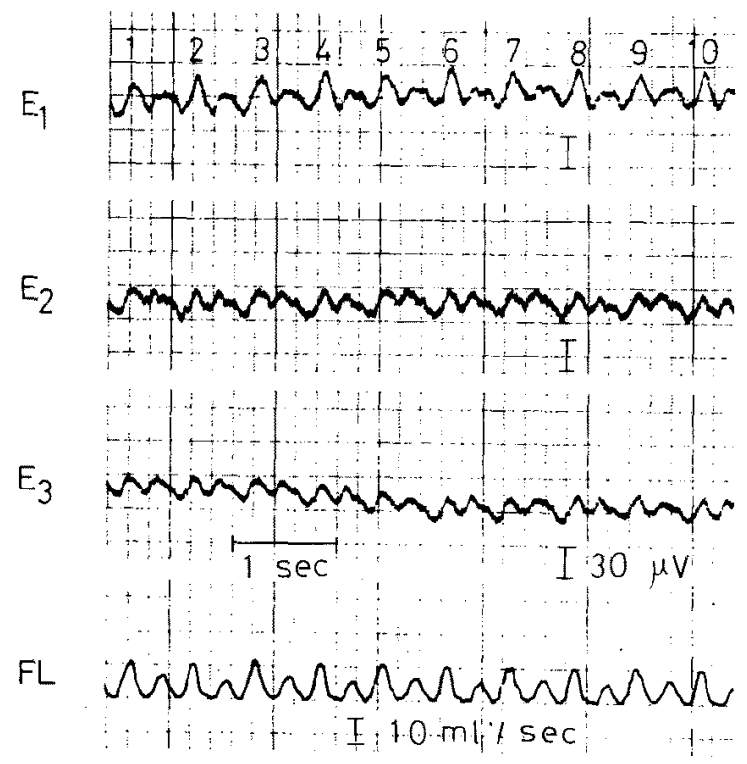

Fig. 5. Flow-induced potentials $E_{z}, E_{2}$, and $E_{z}$ detected with 3 surface electrodes placed at electrode distances of 1,2 , and $3 \mathrm{~cm}$ respectively on the surface; flows (FL) were obtained with an electromagnetic flowmeter; each flow is numbered. 
Table I. Relationship between Calculated and Recorded Flows in a Model Experiment Using Starch Gel

\begin{tabular}{l|l|l|l|l|l|l|l|l|l|l|l|}
\hline No & 1 & 2 & 3 & 4 & 5 & 6 & 7 & 8 & 9 & 10 \\
\hline $\mathrm{E}_{1}$ & 31 & 28 & 34 & 34 & 27 & 34 & 31 & 31 & 28 & 29 \\
$\mathbf{E}_{2}$ & 26 & 21 & 27 & 22 & 21 & 24 & 16 & 24 & 21 & 22 \\
$\mathbf{E}_{3}$ & 13 & 16 & 19 & 16 & 16 & 16 & 16 & 19 & 16 & 19 \\
$\mathrm{Q}_{1}$ & 18 & 16 & 20 & 20 & 16 & 20 & 18 & 18 & 16 & 17 \\
$Q_{2}$ & 19 & 15 & 20 & 16 & 16 & 18 & 12 & 18 & 15 & 16 \\
$Q_{3}$ & 18 & 16 & 19 & 16 & 16 & 16 & 16 & 19 & 16 & 19 \\
$Q_{12}$ & 20 & 15 & 19 & 14 & 15 & 16 & 9 & 17 & 13 & 14 \\
$Q_{13}$ & 12 & 15 & 17 & 17 & 15 & 15 & 15 & 18 & 15 & 19 \\
$Q_{123}$ & 7.5 & 17 & 18 & 16 & 17 & 13 & $*$ & 23 & 17 & 16 \\
$\mathrm{FL}$ & 17 & 16 & 18 & 17 & 18 & 16 & 17 & 16 & 16 & 16
\end{tabular}

No, flow number $; E_{1}, E_{2}, E_{3}, M R G(\mu V)$ at electrode distances of $1,2,3(\mathrm{~cm})$, respectively; $Q_{1}, Q_{2}, Q_{3}$, flows ( $\mathrm{ml} / \mathrm{sec}$ ) calculated by one electrode method from $\mathrm{E}_{1}, \mathrm{E}_{2}, \mathrm{E}_{3}$, respectively; $Q_{12}, Q_{18}$, by two electrode method from $E_{1}, E_{2}$, and $E_{1}, E_{3}$, respectively. $Q_{128}$ by three electrode method from $E_{1}, E_{2}, E_{3}$. A symbol * indicates no answer. FL, flow obtained with an electromagnetic flowmeter.

First of all, the flow coefficient $\mathrm{k}^{\prime}$ was calculated by measuring a conductivity ratio of starch gel to saline: $\sigma_{2} / \sigma_{1}=5 / 6$. That is, $\mathrm{k}^{\prime}=0.29$. Using this coefficient, calculations of the flows numbered were made from the corresponding potentials $\mathrm{E}_{1}, \mathrm{E}_{2}$, and $\mathrm{E}_{3}$ (Fig. 5).

The flows calculated are shown in Table I. There are no significant differences between flows calculated $\left(Q_{1}, Q_{2}, Q_{3}, Q_{12}, Q_{13}\right.$, and $\left.Q_{123}\right)$ and recorded (FL) which were obtained with an electromagnetic flowmeter implanted in the same tube adjacent to the measuring part. $Q_{1}, Q_{i j}$ and $Q_{i j k}$ are flows calculated by the one, two, and three electrode methods, at $\mathrm{i}, \mathrm{j}$, and $\mathrm{k}$ electrode distances. But in the parts symbolized by $*$ there were no answers because of an imaginary number for the second order equation of the given potentials. This may probably originate in noises of the amplifier and/or electrodes.

\section{Animal Experiment}

These 3 methods were brought to use on the lateral side of the femoral artery in the thigh of 8 dogs weighing 7 to $17 \mathrm{Kg}$. In the animal experiment the tissue compositions are so complicated that the flow coefficient can not be calculated theoretically. Then a structural coefficient was introduced which is related to the external shape, internal tissue compositions and their impedance of the body, in the place of the flow coefficient. 
With regard to the structural coefficient, it seems probable that there is no big difference between the individuals with almost the same weight. Once the coefficient has, therefore, been obtained by using, for example, an electromagnetic flowmeter, then blood flows of the subjects under the same measuring conditions may be calculated from the surface induced EMF (MRG) by an equation including the coefficient corresponding to the measuring part.

In the same way as in the case of the model experiment mentioned above (Fig. 4), 4 needle-shaped electrodes were located at electrode distances of $1,2,3$, and $4 \mathrm{~cm}$ on the lateral side of the femoral artery in the thigh of a $12-\mathrm{Kg}$ dog placed in a high magnetic field of $1 \mathrm{~K}$ gauss, to make the axis of the electrode paralleled to the axis of the artery.

An example was given in Fig. 6. The detected potentials $\mathrm{E}_{1}, \mathrm{E}_{2}, \mathrm{E}_{3}$, and $E_{4}$ for each flow numbered were substituted into eq. (3-1), 3 combinations

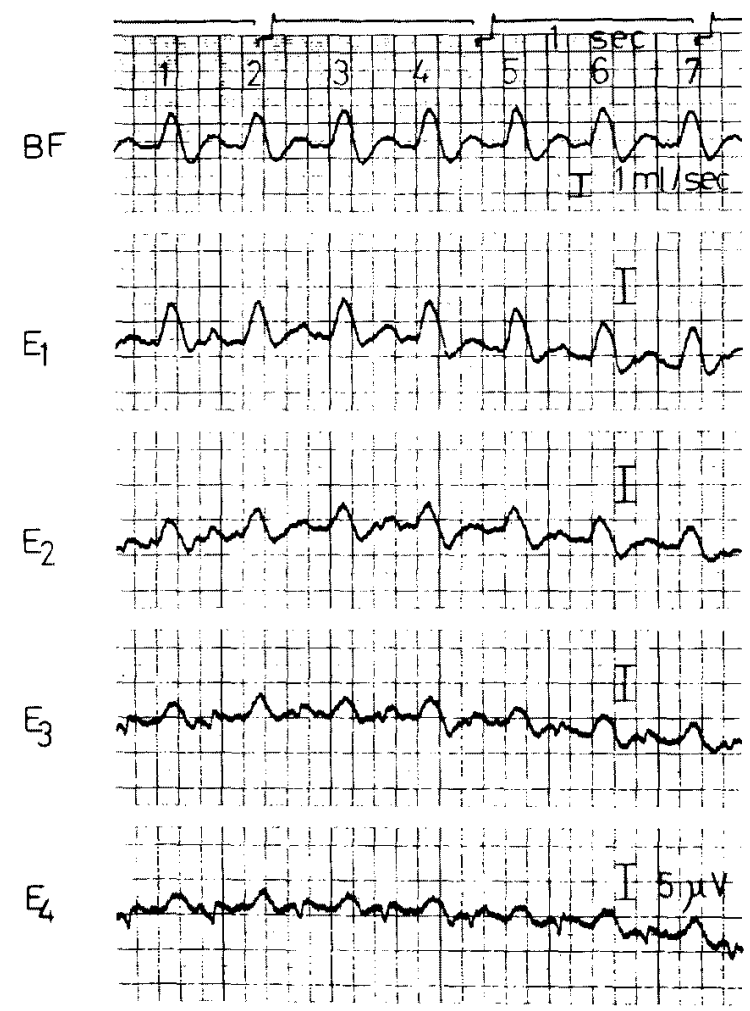

Fig. 6. Flow-induced potentials $E_{1}, E_{2}, E_{3}$, and $E_{4}$ detected with 4 electrodes placed at electrode distances of $1,2,3$, and $4 \mathrm{~cm}$ respectively on the lateral side of the femoral artery in the thigh of a dog weighing $12 \mathrm{Kg}$; blood flows (BF) were obtained with an clcctromagnetic flowmeter; each flow is numbered. 
Table II. Structural Coefficients Obtained by One, Two and Three Electrode Methods from an Experiment on the Lateral Side of the Femaral Artery of a Dog Weighing $12 \mathrm{Kg}$

\begin{tabular}{l|l|l|l|l|l|l|l}
\hline No & 1 & 2 & 3 & 4 & 5 & 6 & 7 \\
\hline $\mathrm{E}_{1}$ & 6.5 & 6.0 & 6.5 & 7.5 & 7.5 & 6.5 & 7.0 \\
$\mathrm{E}_{2}$ & 4.5 & 4.5 & 5.0 & 6.0 & 6.0 & 5.0 & 4.8 \\
$\mathrm{E}_{3}$ & 3.5 & 3.5 & 3.5 & 5.0 & 4.0 & 4.0 & 4.0 \\
$\mathrm{E}_{4}$ & 2.5 & 2.8 & 2.8 & 3.5 & 2.5 & 3.5 & 3.5 \\
$\mathrm{~K}_{1}$ & 0.17 & 0.17 & 0.18 & 0.15 & 0.15 & 0.18 & 0.16 \\
$\mathrm{~K}_{2}$ & 0.19 & 0.18 & 0.18 & 0.15 & 0.15 & 0.18 & 0.18 \\
$\mathrm{~K}_{3}$ & 0.19 & 0.17 & 0.17 & 0.14 & 0.16 & 0.16 & 0.15 \\
$\mathrm{~K}_{4}$ & 0.20 & 0.17 & 0.19 & 0.15 & 0.22 & 0.15 & 0.15 \\
$\mathrm{~K}_{12}$ & 0.21 & 0.17 & 0.19 & 0.15 & 0.15 & 0.19 & 0.20 \\
$\mathrm{~K}_{13}$ & 0.19 & 0.17 & 0.20 & 0.13 & 0.18 & 0.14 & 0.17 \\
$\mathrm{~K}_{14}$ & 0.22 & 0.17 & 0.20 & 0.15 & 0.23 & 0.15 & 0.15 \\
$\mathrm{~K}_{123}$ & 0.11 & 0.14 & 0.22 & 0.16 & 0.22 & 0.13 & $*$ \\
$\mathrm{~K}_{234}$ & 0.31 & 0.16 & 0.18 & 0.23 & 0.31 & $*$ & $*$ \\
$\mathrm{BF}$ & 2.2 & 2.0 & 2.3 & 2.3 & 2.3 & 2.3 & 2.2 \\
\end{tabular}

No, flow number; $E_{1}, E_{2}, E_{3}, E_{4}$, potentials $(\mu V)$ detected with four electrodes at electrode distances of $1,2,3,4(\mathrm{~cm})$ respectively; $\mathrm{K}_{1}, \mathrm{~K}_{2}, \mathrm{~K}_{3}, \mathrm{~K}_{4}$, structural coefficients obtained by one electrode method at electrode distances of $1,2,3,4(\mathrm{~cm})$ respectively; $\mathrm{K}_{12}$, $\mathrm{K}_{13}, \mathrm{~K}_{14}$, structural coefficients by two electrode method at electrode distances of $1,2,1$, 3 , and 1, 4 (cr) respectively; $K_{123}, K_{234}$, structural coefficients by three electrode method at electrode distances of $1,2,3$ and 2,3,4 (cm) respectively; $\mathrm{BF}$, blood flow (ml/sec) obtained with an electromagnetic flowmeter; symbol *, no answer.

of potentials $E_{1} E_{2}, E_{1} E_{3}$, and $E_{1}^{*} E_{4}$ into eq. (3-2), and 2 combinations of potentials $\mathrm{E}_{1} \mathrm{E}_{2} \mathrm{E}_{3}$ and $\mathrm{E}_{2} \mathrm{E}_{3} \mathrm{E}_{4}$ into eq. (3-4). On the other hand, each numbered flow (BF) actually recorded with an electromagnetic flowmeter implanted in the same vessel adjacent to the measuring part was also substituted into eqs. (3-1), (3-2), and (3-4) through eq. (3-5), to give the structural coefficients, as shown in Table II, where $K_{i}, K_{i j}$, and $K_{i j k}$ are the coefficients calculated by the one, two, and three electrode methods, respectively, at $\mathrm{i}$, $\mathrm{j}$, and $\mathrm{k}$ electrode distances. Similar experiments were made on $8 \mathrm{dogs}$ weighing 7 to $17 \mathrm{Kg}$. The mean values for the structural coefficients were obtained as in Fig. 7.

These results show that when the electrode distance covers a range of $3 \mathrm{~cm}$, the curve of the mean values for the coefficients by the one and two electrode methods, are fairly flat, as shown in Fig. 7, and the average of these mean values by the two electrode method is 0.16 . That is, the two electrode method of the coefficient 0.16 , is applicable and convenient for this measurement because of easiness of measuring the electrode distance.

Next, in order to check the coefficient calibrated above, the same type 


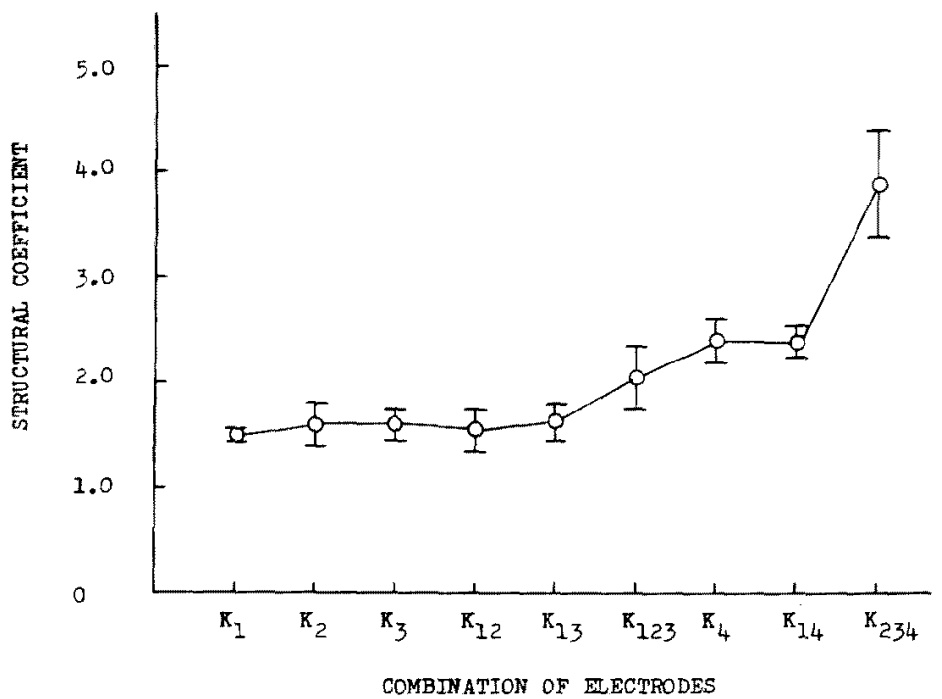

Fig. 7. Mean values of structural coefficients for the lateral side of the femoral artery in the thighs of 8 dogs weighing 7 to $17 \mathrm{Kg} ; \mathrm{K}_{\mathrm{i}}, \mathrm{K}_{\mathrm{ij}}$, and $K_{i, k}$ are the coefficients calculated by one, two, and three electrode methods respectively, at $i, j$, and $k$ electrode distances; vertical axes show standard deviations.

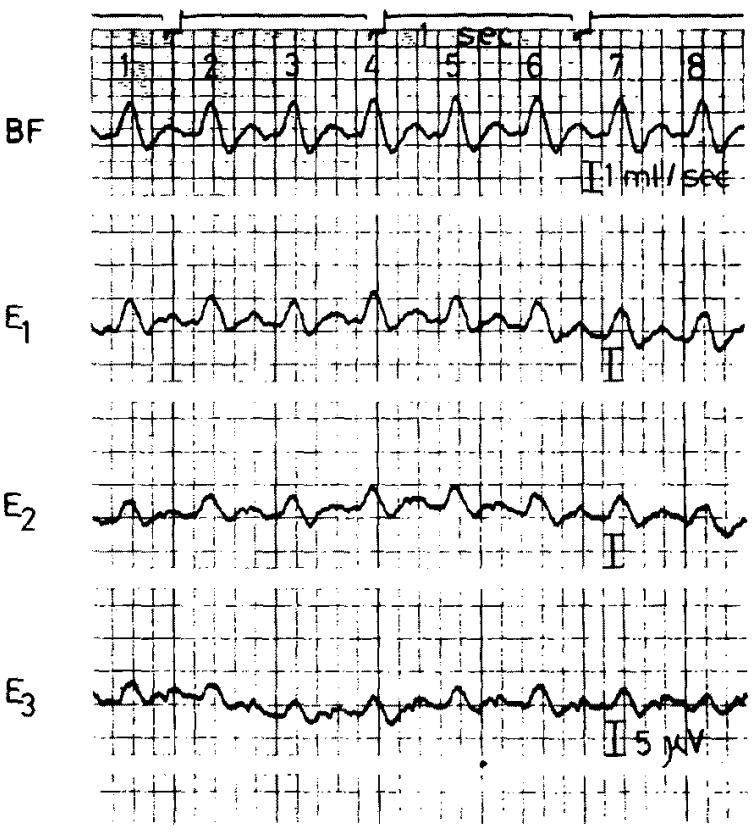

Fig. 8. Flow-induced potentials $\mathrm{E}_{2}, \mathrm{E}_{2}$, and $\mathrm{E}_{3}$ detected with 3 electrodes placed at electrode distances of 1,2 , and $3 \mathrm{~cm}$ respectively on the lateral side of the femoral artery in the thigh of a dog weighing $13 \mathrm{Kg}$; blood flows (BF) were obtained with an electromagnetic flowmeter; each flow is numbered. 
Table III. Relationship between Flows Recored and Calculated by

Two Electrode Method of the Structural Coefficient of 0.16 from an Experiment on the Lateral Side of the Femoral Artery of a Dog Weighing $13 \mathrm{Kg}$

\begin{tabular}{l|l|l|l|l|l|l|l|l|l}
\hline No & 1 & 2 & 3 & 4 & 5 & 6 & 7 & 8 \\
\hline $\mathrm{E}_{1}$ & 4.8 & 4.8 & 5.0 & 5.0 & 5.0 & 6.0 & 6.0 & 5.5 \\
$\mathrm{E}_{2}$ & 3.5 & 3.5 & 4.3 & 4.3 & 4.5 & 4.5 & 4.3 & 4.3 \\
$\mathrm{E}_{3}$ & 3.0 & 3.0 & 3.0 & 3.5 & 3.5 & 4.0 & 4.0 & 3.0 \\
$\mathrm{Q}_{12}$ & 1.3 & 1.3 & 1.8 & 1.8 & 2.0 & 1.7 & 1.6 & 1.7 \\
$\mathrm{Q}_{13}$ & 1.6 & 1.6 & 1.6 & 1.9 & 2.0 & 2.0 & 1.9 & 1.7 \\
$\overline{\mathrm{Q}}_{23}$ & 1.5 & 1.5 & 1.7 & 1.9 & 2.0 & 2.0 & 1.9 & 1.7 \\
$\mathrm{BF}$ & 1.5 & 1.6 & 1.6 & 1.7 & 1.7 & 1.8 & 1.7 & 1.6 \\
$\mathrm{ER}$ & 0 & 6.3 & 6.3 & 12 & 18 & 12 & 12 & 6.3
\end{tabular}

No, flow number $; E_{1}, E_{2}, E_{3}$, potentials $(\mu V)$ detected with three electrodes at electrode distances of 1,2 , and $3(\mathrm{~cm})$ respectively; $Q_{12}, Q_{13}$, flows $(\mathrm{ml} / \mathrm{sec})$ calculated by two electrode method from $E_{1}, E_{2}$, and $E_{1}, E_{3}$ respectively; $\bar{Q}_{23}$, average of $Q_{12}$ and $Q_{13}$; ER, error.

of the experiment was made to place three electrode at electrode distances of 1,2 , and $3 \mathrm{~cm}$ on the lateral side of the femoral artery of dog weighing $13 \mathrm{Kg}$. The detected potentials were given in Fig. 8. The peak-to-peak blood flows numbered were calculated from the corresponding potentials $\mathbf{E}_{\mathbf{1}}$, $\mathbf{E}_{2}$, and $\mathbf{E}_{3}$ by the two electrode method of the coefficient 0.16 , as shown in Table III. There seems to be no great difference between the flows recorded (BF) and calculated $\left(\bar{Q}_{23}\right)$ where $\bar{Q}_{23}$ is the average of $Q_{12}$ and $Q_{13}$. Furthermore, the errors (ER) were within $20 \%$.

\section{Discussion}

We could thus confirm that the theoretical analysis of this measurement (magnetorheography) agreed well with the model experiment and the in situ measurement.

When the skin surface is fairly flat in the vicinity of the vessel measured, an approximate solution (eq. 1) of the flow-induced potential (MRG) was made using Faraday's law and the image theory. The similar consideration was performed elsewhere. ${ }^{8)}$

Frequency transfer functions indicate that by using A-C amplifier, the arterial flow signal of high frequency components is selectively picked up (eq. 2-2), while the venous flow signal of low frequency components and D-C is suppressed (eq. 2-1). From these results and eq. (1), it is clear that MRG is proportional to the arterial flow. This was verified in animal ex- 
periments. ${ }^{2), 3)}$ Furthermore, it was proved in a model experiment that MRG was related only to blood flows but not to the radius of the vessel (Fig. 3), as expressed in eq. (1).

For calculations of flows from MRG, 3 methods were established. In a model experiment using a box-shaped block of starch gel, there were close agreements between flows calculated by these 3 methods and actually recorded (Table I).

In the animal experiment, the tissue compositions are so complicated that the flow coefficient can not be obtained theoretically. Therefore, instead of the flow coefficient $\mathrm{k}^{\prime}$ (eq. 3-1), a structural coefficient was introduced which is related to the external shape, internal tissue compositions and their impedance of the body. A blood flow can be calculated from MRG by an equation including the coefficient.

Experiments were made on the lateral side of the femoral artery in the thighs of $8 \mathrm{dogs}$ weighing 7 to $17 \mathrm{Kg}$. The recorded flows and detected potentials were substituted into eqs. (3-1), (3-2), and (3-4) through eq. (3-5), to give the structural coefficients (Table II and Fig. 7). The mean values of coefficients by the one and two electrode methods were approximately constant, provided that the electrode distance is within $3 \mathrm{~cm}$. When this distance is over $3 \mathrm{~cm}$, the mean values of the coefficients are also greater than 0.20 . This may be because of the approximate derivation of eq. (1). That is, eq. (1) is not satisfied when the electrode distance is over $3 \mathrm{~cm}$.

These results suggest that because of the easiness of measuring the electrode distance, the two electrode method of the structural coefficient 0.16 is applicable to the approximate calculations of blood flows on the lateral side of the femoral artery in the thigh of a dog weighing about $10 \mathrm{Kg}$. Since the structural coefficient 0.16 has been defined as an average of the structural coefficients $K_{12}$ and $K_{13}$, the flow can also be shown as an average $Q_{23}$ of flows $Q_{12}$ and $Q_{13}$ calculated by the two electrode method at electrode distances of 1,2 and $1,3 \mathrm{~cm}$, respectively.

The two electrode method of the coefficient of 0.16 , was brought about to a practical use on the experiment of a dog weighing $13 \mathrm{Kg}$ (Fig. 8). The averages of 2 flows calculated by this method at electrode distances of 1,2 and $1,3 \mathrm{~cm}$, respectively, were approximately equal to the flows actually recorded with an electromagnetic flowmeter, within the error of $20 \%$ (Table III).

For precise calculations of flows by these 3 methods it is necessary to obtain the structural coefficient using a flowmeter which can not be affected by a high magnetic field. The values measured (Tables II and III) may include some amount of errors because we did not completely eliminate 
interference of the high magnetic field with the flow measurement. But the motion artifacts of electrodes were very small and negligible.

\section{ACKNOWLEDGEMENTS}

The authors wish to thank Dr. K. Atsumi, Dr. J. Iriuchijima, Dr. A. Watanabe, Dr. K. Ikeda and Dr. M. Hori for their helpful advices.

\section{APPENDIX}

Provided that blood flow is centrally symmetrical and the radius of the vessel is negligible as compared with the distance (vessel depth) from the surface of the skin to the vessel, blood flow in a magnetic field can be assumed to make a line dipole current source at the center of the vessel. Therefore, the problems considered here is two dimensional.

As a simple example, let us consider a vessel in an infinite plane of the tissue. If both blood and tissue are assumed to be homologous conductors, the potentials due to the dipole of blood flow at arbitrary points are written from Laplace's equation

$$
\begin{aligned}
& V_{i}=\frac{M}{r}+A r \\
& V_{0}=\frac{M}{r}+\frac{B}{r}
\end{aligned}
$$

where $V_{\mathrm{i}} \operatorname{Sin} \theta$ and $V_{\mathbf{0}} \operatorname{Sin} \theta$ show potentials at points in the inside and the outside of the vessel respectively; $M$, a dipole moment; $A$ and $B$, constants; $\theta$, an angle of the vessel between the magnetic field and a measuring point for polar coordinates; $r$, a distance of an examined point from a locating point of the dipole.

In a plane where the dipole due to blood flow is located near a skin surface that is fairy flat, an approximate solution can be made, particularly, for the region in the vicinity of the dipole. This is accomplished by treating the surface as if it were a part of an infinite plane and then replacing the surface effect by an approximate image, ${ }^{5)}$ as shown in Fig. 9.

According to the image theory, a potential at an arbitrary point in an infinite plane is the sum of those due to both actual and image dipoles which have the same moment and are located at the symmetric points with respect to the skin surface each other. Consequently the potentials at arbitrary points were written from eqs. (1) and (2)

$$
\begin{aligned}
& \mathrm{E}_{\mathrm{i}}=\frac{\mathrm{M}}{\mathrm{r}}+\mathrm{Ar}+\frac{\mathrm{M}}{\mathrm{r}^{\prime}}+\frac{\mathrm{B}}{\mathrm{r}^{\prime}} \\
& \mathrm{E}_{\mathrm{o}}=\frac{\mathrm{M}}{\mathrm{r}}+\frac{\mathrm{B}}{\mathrm{r}}+\frac{\mathrm{M}}{\mathrm{r}^{\prime}}+\frac{\mathrm{B}}{\mathrm{r}^{\prime}}
\end{aligned}
$$

where $E_{\mathrm{1}} \operatorname{Sin} \theta$ and $E_{0} \operatorname{Sin} \theta$ are potentials within and without the vessel respectively; $r^{\prime}$, a distance of the examined point $P$ from a locating point of the image dipole: 


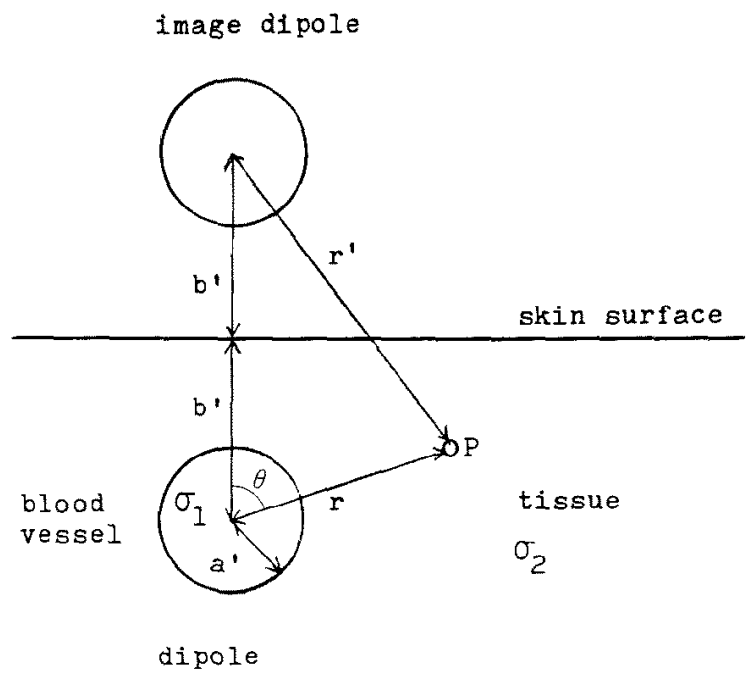

Fig. 9. Relation among dipole, image dipole, and measuring point $\mathrm{P}$; $a^{\prime}$ denotes radius of the vessel; $b^{\prime}$, vessel depth; $r$ and $r^{\prime}$, distances of measuring point $\mathrm{P}$ from the dipole and the image one, respectively; $\sigma_{1}$ and $\sigma_{2}$, conductivities of blood and tissue, respectively.

$r^{\prime}=\left(r^{2}+4 b^{\prime 2}-4 r b^{\prime} \operatorname{Cos} \theta\right)$ where $b^{\prime}$ is a vessel depth.

Boundary conditions must be satisfied on the borderline between blood and vessel wall. Considering the boundary conditions, from eqs. (A-3) and (A-4) we get; $E_{\mathrm{i}}=E_{0}$ and $\sigma_{1} \partial E_{\mathrm{i}} / \partial r=\sigma_{2} \partial E_{0} / \partial r$ for $r=a^{\prime}$ where $\sigma_{1}$ and $\sigma_{2}$ are conductivities of blood and tissue, respectively; $a^{\prime}$ is the internal radius of the vessel. Then we obtain

$$
\mathrm{B}=\frac{\sigma_{1}-\sigma_{2}}{\sigma_{1}+\sigma_{2}}
$$

Since the radius $a^{\prime}$ is negligible as compared with the vessel depth $b^{\prime}: 1 \gg a^{\prime}\left\langle b^{\prime}\right.$, as mentioned above.

Rearranging eq. (A-4) by substituting eq. (A-5) into eq. (A-4) we get a potential $E$ at a skin surface point $p(r, \theta)$

$$
\mathrm{E}=\frac{4 \sigma_{1}}{\sigma_{1}+\sigma_{2}} \frac{\mathrm{M}}{\mathrm{r}} \sin \theta
$$

$M$ is obtained in such a way as follows. The potential induced by a flow at a point on the borderline between blood and vessel wall in the case of an electromagnetic flowmeter is equal to that at the same point in this magnetorheographic measurcment where the boundary conditions:

$\mathrm{V}_{\mathrm{i}}=\mathrm{V}_{\mathrm{o}}$ and $\sigma_{1} \delta \mathrm{V}_{\mathrm{i}} / \hat{o} \mathrm{r}=\sigma_{2} \delta \mathrm{V}_{\mathrm{o}} / \hat{\mathrm{r}}$ for $\mathrm{r}=\mathrm{a}^{\prime}$ and $\sigma_{2}=0$, namely $\mathrm{V}_{\mathrm{i}}=2 \mathrm{M} / \mathrm{a}^{\prime}$, is satisfied ${ }^{\prime \prime}$. Then we get

$$
\frac{20 \mathrm{H}}{\mathrm{a}^{\prime}} \int_{0}^{a \prime} \mathrm{f}(\mathrm{r}) \mathrm{rdr}=\frac{10}{\mathrm{a}^{\prime}} \mathrm{HQ}=\frac{2 \mathrm{M}}{\mathrm{a}^{\prime}}
$$


where $f(r)$ is local flow velocity (ml/sec); $H$, a field intensity ( $K$ gauss); $Q$, a flow $(\mathrm{ml} / \mathrm{sec})$. Hence we get

$$
\mathrm{M}=\frac{5}{\pi} \mathrm{HQ}
$$

Substituting eq. (A-8) into eq. (A-6) we get

$$
\mathrm{E}=\frac{20}{\pi} \frac{\sigma_{1}}{\sigma_{1}+\sigma_{2}} \frac{\mathrm{HQ}}{\mathrm{r}} \sin \theta
$$

Transforming eq. (A-9) to the rectangular coordinates where $x=r \operatorname{Sin} \theta, y-y_{1}=r$ $\operatorname{Cos} \theta$, as shown in Fig. 1, we get a potential $E$ at a skin surface point $P(x, 0)$ regardless the radius of the vessel

$$
\mathrm{E}=\frac{20}{\pi} \frac{\sigma_{1}}{\sigma_{1}+\sigma_{2}} \mathrm{HQ} \frac{\mathrm{x}}{\mathrm{x}_{2}+\mathrm{y}_{1}^{2}}
$$

where $y_{1}$ is a vessel depth; the potential is expressed in microvolts.

\section{REFERENCES}

1. Kolin A: An alternating feld induction flow meter of high sensitivity. Rev Sci Instr 16: 109,1945

2. Okai O: Magnetorheography. Doctral Thesis for Postgraduate School, Faculty of Medicine, University of Tokyo, 1969

3. Okai O: Magnetorheography. Non-bleeding measurement of blood flow. J Appl Physiol 30: 564,1971

4. Okai O, Togawa T, Oshima M: Magnetorheography. Observation of blood flow EMF in static magnetic field by surface electrodes. Digest of 7 th International Conference on Medical and Biological Engineering. 212, 1967

5. Plosey R: Current dipole images and reference potentials. IEEE Trans Biomed Electronics BME-10: 3, 1963

6. Togawa $\mathrm{T}$, Okai $\mathrm{O}$, Oshima $\mathrm{M}$ : Observation of blood flow EMF in externally applied strong magnetic field by surface electrodes. Med \& Biol Engng 5: 169, 1967

7. Yamano E, Kanai $H$, Nakayama $K$, Kawamura N: Transcutaneous blood flow measurement by electromagnetic induction. Jap J Med \& Biol Engng 8: 444, 1970

8. Kanai H, Yamano E, Nakayama K, Kawamura N, Furuhata H: Transcutaneous blood flow measurement by electromagnetic induction. IEEE Trans Biomed Engng BME-21: 144, 1974 